\title{
Mesenchymal stromal cells as vehicles of tetravalent bispecific Tandab (CD3/CD19) for the treatment of B cell lymphoma combined with IDO pathway inhibitor D-1-methyl-tryptophan
}

Xiaolong Zhang ${ }^{1}$, Yuanyuan Yang ${ }^{1}$, Leisheng Zhang ${ }^{1}$, Yang Lu' , Qing Zhang ${ }^{1}$, Dongmei Fan', Yizhi Zhang ${ }^{2}$, Yanjun Zhang ${ }^{1 *}$, Zhou Ye $\mathrm{e}^{2^{*}}$ and Dongsheng Xiong ${ }^{1 *}$

\begin{abstract}
Background: Although blinatumomab, a bispecific T cell engaging antibody, exhibits high clinical response rates in patients with relapsed or refractory B-precursor acute lymphoblastic leukemia (B-ALL) and B cell non-Hodgkin's lymphoma (B-NHL), it still has some limitations because of its short half-life. Mesenchymal stromal cells (MSCs) represent an attractive approach for delivery of therapeutic agents to cancer sites owing to their tropism towards tumors, but their immunosuppression capabilities, especially induced by indoleamine 2,3-dioxygenase (IDO), should also be taken into consideration.

Methods: Human umbilical cord-derived MSCs (UC-MSCs) were genetically modified to secrete Tandab (CD3/CD19), a tetravalent bispecific tandem diabody with two binding sites for CD3 and two for CD19. The tropism of MSCs towards Raji cells in vitro was determined by migration assays, and the homing property of MSCs in vivo was analyzed with firefly luciferase-labeled MSCs (MSC-Luc) by bioluminescent imaging (BLI). The cytotoxicity of T cells induced by MSCsecreting Tandab (CD3/CD19) was detected in vitro and in vivo in combination with D-1-methyl-tryptophan (D-1MT), an IDO pathway inhibitor.

Results: The purified Tandab (CD3/CD19) was functional with high-binding capability both for CD3-positive cells and CD19-positive cells and was able to induce specific lysis of CD19-positive cell lines (Raji, Daudi, and BJAB) in the presence of T cells. Additionally, results from co-culture killing experiments demonstrated that Tandab (CD3/CD19) secreted from MSCs was also effective. Then, we confirmed that D-1MT could enhance the cytotoxicity of T cells triggered by MSC-Tandab through reversing T cell anergy with down-regulation of CD98 and Jumonji and restoring the proliferation capacity of T cells. Furthermore, MSC-Luc could selectively migrate to tumor site in a BALB/C nude mouse model with Raji cells. And mice injected with MSC-Tandab in combination with D-1MT significantly inhibited the tumor growth.
\end{abstract}

Conclusions: These results suggest that UC-MSCs releasing Tandab (CD3/CD19) is an efficient therapeutic tool for the treatment of B cell lymphoma when combined with D-1MT.

Keywords: MSCs, Bispecific antibodies, CD3, CD19, D-1-methyl-tryptophan, B cell lymphoma

\footnotetext{
*Correspondence: junjunfriend@126.com; yezhou126@126.com;

dsxiong@ihcams.ac.cn

'State Key Laboratory of Experimental Hematology, Institute of Hematology and Hospital of Blood Diseases, Chinese Academy of Medical Science and Peking Union Medical College, Tianjin 300020, People's Republic of China ${ }^{2}$ Central Hospital of Karamay, Karamay, Xinjiang 834000, People's Republic of China
} 


\section{Background}

During the past two decades, therapeutic antibodies have started to make major contributions to the treatment of B cell malignancies. In 1997, rituximab (a genetically engineered monoclonal chimeric antibody directed against the CD20 B cell antigen) was approved by the US Food and Drug Administration (FDA) for the indication of follicular lymphoma and low-grade B cell nonHodgkin's lymphoma (B-NHL) [1]. However, cancer relapse and metastasis often occurred after medical intervention with CD20-based therapy in patients with follicular lymphoma and acute lymphocytic leukemia [2]. Thus, it is absolutely critical to develop new therapeutic regimens to overcome these challenges.

$\mathrm{T}$ cells are the most potent tumor-killing effector cells, but they cannot be recruited by conventional antibodies. However, several bispecific antibodies (bsAbs) that recruit $\mathrm{T}$ cells have been developed [3], which may have the potential to circumvent this problem. To date, the most promising for the therapeutic application of this approach is blinatumomab, a tandem single-chain variable fragment (scFv) bsAb in a bispecific $\mathrm{T}$ cell engager (BiTE) format targeting CD19/CD3, which was approved by the FDA for the treatment of B-precursor acute lymphoblastic leukemia (B-ALL) [4]. Although blinatumomab has impressive efficacy in the clinic and exhibits high clinical response rates in patients with relapsed or refractory B-ALL and B-NHL $[5,6]$, it still has some limitations due to its low molecular weight $(\sim 55 \mathrm{kDa})$, which is below the glomerular filtration threshold [6]. Therefore, blinatumomab is administered over a 28-day continuous infusion using a mini-pump in order to maintain steady drug concentration [6], which results in inconvenience for patients and an increased possibility of treatment-related adverse event. To overcome the drawback of short half-life, Kipriyanov and his colleagues [7] firstly designed a tetravalent bispecific tandem diabody (TandAb) with two binding sites for CD3 and two for CD19. Due to the TandAb's large molecular weight $(\sim 105 \mathrm{kDa})$, it is not subject to glomerular filtration. In addition, the molecule exhibits stability properties with a half-life ranging from 18.4 to $22.9 \mathrm{~h}$ after intravenous administration in mice [8].

Most of therapeutic antibodies are administrated by intravenous infusion, which results in a handful of antibodies that can reach the tumor sites. Thus, introducing an efficient and targeted delivery system for these therapeutic antibodies may enhance the efficacy of treatment for tumors, especially for minimal residual diseases (MRD) [9]. Mesenchymal stromal cells (MSCs) are attractive cellular vehicles for the therapy of malignant diseases as they have the ability to migrate into tumors and track microscopic metastasis [10, 11]. We have previously employed human umbilical cord-derived MSCs
(UC-MSCs) as carriers for gene therapy $[12,13]$ because UC-MSCs are easier to isolate and expand, and the harvesting procedure is more consistent and yields a greater number of relevant cells than other adult and feta tissues [14]. These characteristics indicate that UC-MSC is a promising targeted delivery system with anticancer agents for a variety of cancers.

However, a study from Ribeiro and his colleagues shows that MSCs derived from different tissues possess different immunosuppression capabilities and their action varies with the immune cell type [15]. Thus, MSCs are actually a double-edged sword when employed as carriers with agents triggering cytotoxicity of $\mathrm{T}$ cells for tumors. Although the exact mechanisms of MSCmediated immunosuppression are still debated, many different factors are believed to be involved. It has been revealed that indoleamine 2,3-dioxygenase (IDO), the first and rate-limiting enzyme in the degradation of tryptophan [16], plays a role in human MSCs to regulate immunity in tumor microenvironment [17]. By depleting tryptophan locally and accumulation of its metabolites such as kynurenine and quinolinic acid, which could interact the aryl hydrocarbon receptor (AHR) on T cells, IDO seems to block the proliferation of $\mathrm{T}$ cells and inhibit $\mathrm{T}$ cell activity $[18,19]$. Because IDO is induced by inflammatory cytokines such as interferon- $\gamma$ (IFN- $\gamma$ ) which act through JAK-STAT signaling pathways on interferon stimulatory response elements (ISRE) and $\gamma$-activating sequences (GAS) in the IDO promoter [20], its expression is thought to be an endogenous feedback mechanism controlling excessive immune response [21].

In this study, we employed the TandAb platform to construct a new tandem tetravalent antibody targeting both CD3 and CD19 (Tandab (CD3/CD19)) using our own DNA sequences of scFvs. Then, we exploited the feasibility and efficacy of using genetically modified UCMSCs which constitutively secreted Tandab (CD3/ CD19) (MSC-Tandab) for the treatment of human B cell lymphoma. Our results showed that MSC-Tandab could induce specific lysis of CD19-positive Raji cells in the presence of $\mathrm{T}$ cells in vitro and reduce xenograft tumor growth in vivo in combination with D-1-methyltryptophan (D-1MT), an IDO pathway inhibitor [22].

\section{Methods}

\section{Cell lines and cell culture}

Human embryonic kidney cell-derived 293T cells (kindly provided by Professor Cheng Tao, PUMC) were maintained in DMEM (Invitrogen, USA) supplemented with $2 \mathrm{mM}$ L-glutamine, $100 \mathrm{U} / \mathrm{mL}$ penicillin (Gibco, USA), $100 \mu \mathrm{g} / \mathrm{mL}$ streptomycin (Gibco, USA) and 10\% FBS (Gibco, USA). The human acute T cell leukemia cell line Jurkat, human chronic myelogenous leukemia cell line 
K562, and human B cell lymphoma cell lines Raji, Daudi, and BJAB (Institute of Hematology and Blood Diseases Hospital, Chinese Academy of Medical Science and Peking Union Medical College, Tianjin, China) were grown in RPMI-1640 medium (Invitrogen, USA) supplemented with $2 \mathrm{mM}$ L-glutamine, $100 \mathrm{U} / \mathrm{mL}$ penicillin (Gibco, USA), $100 \mu \mathrm{g} / \mathrm{mL}$ streptomycin (Gibco, USA), and $10 \% \mathrm{FBS}$. The cells were incubated at $37{ }^{\circ} \mathrm{C}$ in a humidified atmosphere containing $5 \% \mathrm{CO}_{2}$.

\section{PBMC isolation}

With informed consent, human peripheral blood mononuclear cells (PBMCs) were isolated from healthy volunteers. Details are provided in the Additional file 1.

\section{Construction of lentiviral expression vectors}

Plasmids pLH-T3a*-19a (containing a cysteine at position Ser-100 of $\mathrm{V}_{\mathrm{L}} 3$ ) and pLH-19a-T3a* (containing a cysteine at position Gly-44 of $\mathrm{V}_{\mathrm{H}} 3$ ) encoding for hybrid $\mathrm{V}_{\mathrm{L}} 3-\mathrm{V}_{\mathrm{H}} 19$ and $\mathrm{V}_{\mathrm{L}} 19-\mathrm{V}_{\mathrm{H}} 3$ scFvs [23], respectively, were used for assembly of Tandab (CD3/CD19) genes. See details in Additional file 1.

\section{Expression and purification of Tandab (CD3/CD19)}

293T cells were transfected with pLentiR-Tandab (CD3/CD19) or pLentiR-EV using Lipofectamine 2000 (Invitrogen, USA) according to the manufacturer's protocol. After $48 \mathrm{~h}$ of transfection, supernatants were collected by centrifugation at $500 \times \mathrm{g}$ for $10 \mathrm{~min}$ at $4{ }^{\circ} \mathrm{C}$ to clear $293 \mathrm{~T}$ cells. The soluble antibodies in the supernatants were purified by $6 \times$ His-tag affinity chromatography (GE Healthcare, Sweden) according to the manufacturer's instruction. The purified preparations were quantified with His-tag ELISA detection kit (GenScript, USA) and were used for cell-binding assays and cytotoxicity assays in vitro. In addition, the unpurified or purified Tandab (CD3/CD19) were verified by Western blot analysis.

\section{Cell-binding assay}

The CD19-positive cell lines Raji, Daudi, and BJAB and the CD3-positive cell line Jurkat were employed for analysis of binding activity of Tandab (CD3CD19) by flow cytometry (LSRII, Becton Dickinson Bioscience, San Jose, CA). The CD19- and CD3-negative K562 cells were served as negative control. See details in Additional file 1.

\section{Cytotoxicity assay}

All cytotoxicity assays were performed with PBMC effector cells. And PBMCs were pre-activated with $50 \mathrm{IU} / \mathrm{mL}$ IL-2 for 3 days before cytotoxicity assays. $\mathrm{CD}_{1}{ }^{+}$cells (Raji, Daudi, and BJAB) and $\mathrm{CD} 19^{-}$cells (K562) were prepared as target cells. The specific lysis of target cells was detected by LDH release assay according to the manufacturer's protocol. See details in Additional file 1.

\section{MSCs preparation}

MSCs were isolated from human umbilical cord Wharton's jelly (WJ) as previous described [24]. MSCs were cultured at a density of $8 \times 10^{3} \mathrm{cell} / \mathrm{cm}^{2}$ in DF-12 medium (Invitrogen, USA) supplemented with $2 \mathrm{mM}$ L-glutamine and 10\% FBS (Gibco, USA). When cells reached $80 \sim 90 \%$ confluence, they were detached using a $0.125 \%$ trypsin/1 mM EDTA solution and re-seeded using the same growth medium for subsequent passages. For all experiments, early passages MSCs (3P to $5 \mathrm{P}$ ) were used.

\section{Production of lentivirus}

The lentiviral particles carrying Tandab (CD3/CD19) gene were packaged according to the SBI's protocol. See details in Additional file 1.

\section{Transduction of MSCs and viability of transduced MSCs}

The transduction of MSCs was performed as previously reported [12]. And viability of transduced MSCs was detected by MTT assays. See details in Additional file 1.

\section{Immunophenotype profile and tri-lineage differentiation of MSCs}

MSCs and transduced MSCs (including MSC-EV and MSC-Tandab) were trypsinized (0.125\% trypsin-EDTA) and washed twice with PBS, then incubated with APClabeled anti-human CD73, CD90, CD105, CD14, CD19, CD34, CD45, and HLA-DR (all from BD Biosciences) for $30 \mathrm{~min}$. After washing with PBS, the expression level of these molecules was determined by flow cytometry.

To test the in vitro differentiation ability, MSCs or transduced MSCs were cultured in adipogenic, osteogenic, and chondrogenic differentiation medium, respectively. For adipogenic differentiation, the MSCs were maintained in medium containing $1 \mathrm{mM}$ dexamethasone, $500 \mu \mathrm{M}$ IBMX, $10 \mu \mathrm{g} / \mathrm{mL}$ insulin, and $60 \mu \mathrm{M}$ indomethacin (all from Sigma). Three weeks later, the cells were fixed and stained with Oil Red O (Sigma). For osteogenic differentiation, cells were cultured in IMDM (Gibco) supplemented with 10\% FBS, $100 \mathrm{nM}$ dexamethasone, $50 \mu \mathrm{g} / \mathrm{mL}$ ascorbic acid, and $10 \mu \mathrm{M} \beta$ glycerophosphate (all from Sigma) for about 3 weeks. At the end of incubation, the cells were assayed by Alizarin Red S (Sigma) staining for calcium deposition. To induce chondrogenic differentiation, MSCs were maintained in medium with $100 \mathrm{nM}$ dexamethasone, $50 \mu \mathrm{g} / \mathrm{mL}$ ascorbic acid, $40 \mu \mathrm{g} / \mathrm{mL}$ proline, $10 \mathrm{ng} / \mathrm{mL}$ TGF- $\beta_{3}, 2 \mathrm{mM}$ ITS, $53.5 \mu \mathrm{g} / \mathrm{mL}$ lindeic acid, and $12.5 \mu \mathrm{g} / \mathrm{mL}$ BSA (all from Sigma). Three weeks later, the cultured cells were stained with Alcian Blue (Sigma). In addition, RNA was 
isolated from cells after the induction, and the expression level of differentiation-related gene was determined by real-time PCR on an ABI Prism 7500 detection system (Applied Biosystems, USA). And primers used for real-time PCR were summarized in Table 1.

\section{In vitro and in vivo MSC migration assay}

In vitro and in vivo MSCs migration assays were performed as previously reported [12, 25]. Details are provided in Additional file 1.

\section{In vitro co-culture killing experiments}

To assess the bioactivity of Tandab (CD3/CD19) secreted by MSCs, a co-culture system using transwell plates with $0.4-\mu \mathrm{m}$-pore membrane was established. The specific lysis of target cells was determined by FACS analysis according to the "calcein-loss" method [26]. Briefly, MSC-Tandab, MSC-EV, or MSCs were seeded into 6-well culture plates at a density of $1 \times 10^{5}$ cells per well and incubated for $72 \mathrm{~h}$. Then, Raji cells labeled with calcein-AM (Sigma, USA) and pre-activated PBMCs were added to the equilibrated inserts at an E:T ratio of 10:1. After co-cultured for $24 \mathrm{~h}$, the cells in the inserts were harvested to be detected by flow cytometry. The expression of activation surface markers CD69 and CD25 of T cells was detected by flow cytometry in the same conditions of co-culture system with unlabeled

Table 1 Primer sequences for genes in real-time PCR

\begin{tabular}{ll}
\hline Gene & Primer sequence (from 5' to 3') $^{\prime}$ \\
\hline PPAR-r & Forward: GCTGGCCTCCTTGATGATA \\
ADIPOQ & Forward: TGGTCCTAAGGGGGAGACATCG \\
& Reverse: TGGAATTACCAGTGGAGCC \\
RUNX2 & Forward: CTCACTACCACACCTACCTG \\
& Reverse: TCAATATGGTCGCCAAACAGATTC \\
BGLAP & Forward: GGCGCTACCTGTATCAATGG \\
& Reverse: TCAGCCAACTCGTCACAGTC \\
SOX9 & Forward: AATGGAGCAGCGAAATCAAC \\
& Reverse: CAGAGAGATTTAGCACACTGATC \\
COL2A1 & Forward: GGCAATAGCAGGTTCACGTACA \\
& Reverse: CGATAACAGTCTTGCCCCACTT \\
IDO & Forward: GCCCTTCAAGTGTTCACCAA \\
& Reverse: CCAGCCAGACAAATATATGCGA \\
& Forward: GCTGCTGCTCTTCTGGCTC \\
CD98 & Reverse: GCCAGTGGCATTCAAATAC \\
& Forward: GCTCAGGACTTACGGAAACA \\
Jumonji & Reverse: TGTGGTTGACAGCGGAACTG \\
& Forward: GGTCTTACTCCTTGGAGGCCATGT \\
GAPDH & Reverse: ACCTAACTACATGGTTACATGTT \\
\hline
\end{tabular}

Raji cells. And the supernatants in the inserts were collected for the assay of cytokines produced in the coculture system, including IL-2, IFN- $\gamma$, and TNF- $\alpha$ using ELISA kits (R\&D system, USA).

Because of expression of IDO in MSCs induced by IFN- $\gamma$ in the co-culture system, we performed a coculture experiment for a longer time in absence or presence of D-1MT (Sigma-Aldrich, USA), which is an IDO pathway inhibitor. As mentioned above, MSC-Tandab, pre-activated PBMCs, and Raji cells were added respectively into the co-culture system with or without $1 \mathrm{mM}$ D-1MT and co-cultured for 24, 48, and $72 \mathrm{~h}$. Cells in the upper chamber were harvested at the indicated time points. And the residual Raji cells were determined by flow cytometry with FITC-conjugated anti-CD19 antibodies. The supernatants were harvested for the measure of kynurenine, a metabolite of tryptophan in the IDO pathway. Repetitive wells were set for detection of CD98 and Jumonji in the messenger RNA (mRNA) level at different time points $(24,48$, and $72 \mathrm{~h}$ ) by real-time $\mathrm{PCR}$. In addition, the proliferation of $\mathrm{T}$ cells in the coculture system was detected by BrdU Flow Kit (BD Bioscences). Details are provided in Additional file 1.

\section{Inducible expression of IDO in MSCs}

MSCs were seeded into 6-well culture plates at a density of $1 \times 10^{5}$ cells per well in the absence or presence of $20 \mathrm{ng} / \mathrm{mL}$ of recombinant human IFN- $\gamma$ (R\&D system, USA). After incubation for $48 \mathrm{~h}$, expression of IDO in the level of mRNA or protein upon treatment with IFN- $\gamma$ was verified by real-time PCR and Western blot analysis.

\section{Detection of kynurenine}

Because IDO catalyzes the metabolism of tryptophan in the kynurenine pathway, the activity of IDO was determined by spectrophotometric assay for kynurenine [17] in supernatants from co-culture system and cultures of MSCs with or without exogenous IFN- $\gamma$ stimulation. See details in Additional file 1.

\section{Cell viability assay}

Cell viability assay was performed by MTT assay (SigmaAldrich, USA). See details in Additional file 1.

\section{Western blot analysis}

The expression of specific protein was detected by Western blot analysis. See details in Additional file 1.

\section{Real-time PCR}

Total RNA was extracted from corresponding cells using Trizol reagent (Invitrogen, USA) following the manufacturer's protocol. The complementary DNA (cDNA) was generated using OligdT primers and M-MLV reverse 
transcriptase (Invitrogen, USA) with $2 \mu \mathrm{g}$ total RNA. Real-time PCR was performed using ABI Prism 7500 real-time PCR system (Applied Biosystems, USA), in combination with SYBR Green (Takara, Dalian, China). Specific primers for each gene (Table 1) were selected using Primer Express (Applied Biosystems, USA). Relative transcript expression was normalized to that of GAPDH mRNA.

\section{Growth inhibition of B cell lymphoma xenografts in vivo} All animal studies were performed in accordance with the guidelines under the Animal Ethics Committee of the Institute of Hematology and Hospital of Blood Diseases, Chinese Academy of Medical Sciences and Peking Union Medical College. Raji cells $\left(2 \times 10^{7}\right.$ cells per mouse) were implanted subcutaneously into the right flank of each BALB/c nude mice (female, 5-6 weeks of age; PUMC, China) 1 day after the application of total body irradiation (300 cGy). One week later when tumor size reached $100-200 \mathrm{~mm}^{3}$, the mice were treated intravenously with MSC-Tandab and pre-activated PBMCs and D-1MT in the drinking water. Then, the mice were sacrificed for analysis of organ damages in the indicated time. In tumor therapy experiments, the mice were randomly divided into six groups (five mice for each group) as follows: (a) $\mathrm{MSC}+\mathrm{PBMC}$; (b) MSC-EV + PBMC; (c) MSC-Tandab + PBMC; (d) $\mathrm{MSC}+\mathrm{PBMC}+\mathrm{D}-1 \mathrm{MT}$; (e) MSC-EV + PBMC + D-1MT; (f) MSC-Tandab + PBMC + D-1MT. MSCs $\left(1 \times 10^{6}\right.$ cells per mouse) were injected intravenously at day 0 , followed by pre-activated PBMCs $\left(5 \times 10^{6}\right.$ cells per mouse $)$ via the vein 2 days later, every 7 days for 2 weeks. The mice were treated with or without D-1MT $(2 \mathrm{mg} / \mathrm{mL}$ in drinking water) from the beginning to the end of the treatment. At day 21 after treatment started, the mice were sacrificed by cervical dislocation under anesthesia. Then, tumor tissues were harvested and weighted for treatment evaluation.

\section{Statistical analysis}

Data are represented as mean \pm SD. Statistical analysis was performed using GraphPad Prism 6 or Microsoft Excel software. Significance was assayed by an unpaired two-tailed Student $t$ test or ANOVA ( ${ }^{*} P<0.05$, ${ }^{* *} P<0.01$, ***: $P<0.001)$.

\section{Results}

\section{Design and production of Tandab (CD3/CD19)}

The concept of dimerizing scFv fragments having a short peptide linker between the domains to create two antigen-binding sites pointing in opposite directions [27] was extended to single-chain molecules containing four antibody variable domains [8]. We previously constructed a CD3 $\times$ CD19 bispecific diabody comprising two hybrid $\mathrm{scFv}$ fragments: an anti-human $\mathrm{CD} 3 \mathrm{~V}_{\mathrm{L}}$ domain connected to an anti-human CD19 $\mathrm{V}_{\mathrm{H}}$ domain by a short linker peptide (Gly-Gly-Gly-Gly-Ser, G4S) and anti-CD19 $\mathrm{V}_{\mathrm{L}}$ connected to an anti-CD3 $\mathrm{V}_{\mathrm{H}}$ by a similar linker [23]. We have now fused these hybrid svFvs into a single-chain polypeptide using a long $(\mathrm{G} 4 \mathrm{~S})_{3}$ linker (Fig. 1a). The fusion gene fragment $\mathrm{V}_{\mathrm{L}} 3-\mathrm{V}_{\mathrm{H}} 19-\mathrm{V}_{\mathrm{L}} 19-\mathrm{V}_{\mathrm{H}} 3$ was inserted into a lentiviral expression vector under the control of constitutively active SV40 promoter. A hexahistidine-tag ( His $_{6}$-tag) was added to the carboxyl terminus of the construct to aid in the detection and purification of the products. Upon expression, two polypeptide gene products dimerize in a head-to-tail fashion. These active homodimers (Tandab (CD3/CD19)) have two binding sites for CD3 and two for CD19 (Fig. 1b). To verify the formation of this gene products, Tandab (CD3/CD19) was firstly expressed in adherent 293T cells. In the culture supernatant, protein with a molecular weight of $106 \mathrm{kDa}$ was detected on non-reducing status, and a $53-\mathrm{kDa}$ protein was also detected under the reducing status (Fig. 1c). As shown in Fig. 1d, Tandab (CD3/CD19) maintained a dimer formation after purification in the natural state. Because of the interactions between molecules, a band at $212 \mathrm{kDa}$ was exposed, which indicated that part of the products were tetramers.

\section{Tandab (CD3/CD19) binds specifically both to its target antigens}

Binding specificities of the Tandab (CD3/CD19) were shown by flow cytometry analysis on a number of different CD19-positive B cell lymphoma cell lines, including Raji, Daudi, and BJAB, and CD3-positive Jurkat cells. No binding was detectable on chronic myelogenous leukemia cell line K562, which expresses neither CD19 nor CD3 (Fig. 1e). Furthermore, Tandab (CD3/CD19) was able to significantly prevent parental monoclonal antibodies HIT19a [28] or HIT3a [29] from binding to Raji, BJAB, Daudi, or Jurkat cells, respectively, in a competitive binding assay (Fig. 1f).

\section{Tandab (CD3/CD19) mediates specific target cell lysis in combination with PBMCs}

To investigate the tumor lysis mediated by Tandab (CD3/CD19) in the presence of activated human T cells, a nonradioactive cytotoxicity assay was performed. For this purpose, a panel of CD19-positive cell lines of B cell lymphoma was used as targets with PBMC: target cell ratios ranging from 20:1 to 1:1. Tandab (CD3/CD19) was added at a concentration of $8 \mathrm{pmol} / \mathrm{mL}$ and after an 8 -h reaction time, the target cell lysis was measured by LDH release described previously [30]. Although CD19negative K562 cells were also lysed (less than 30\% at the highest E:T ratio), which may result from aspecific effects, Tandab (CD3/CD19) produced significant specific 
a

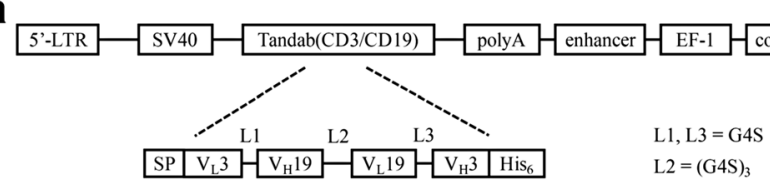

b
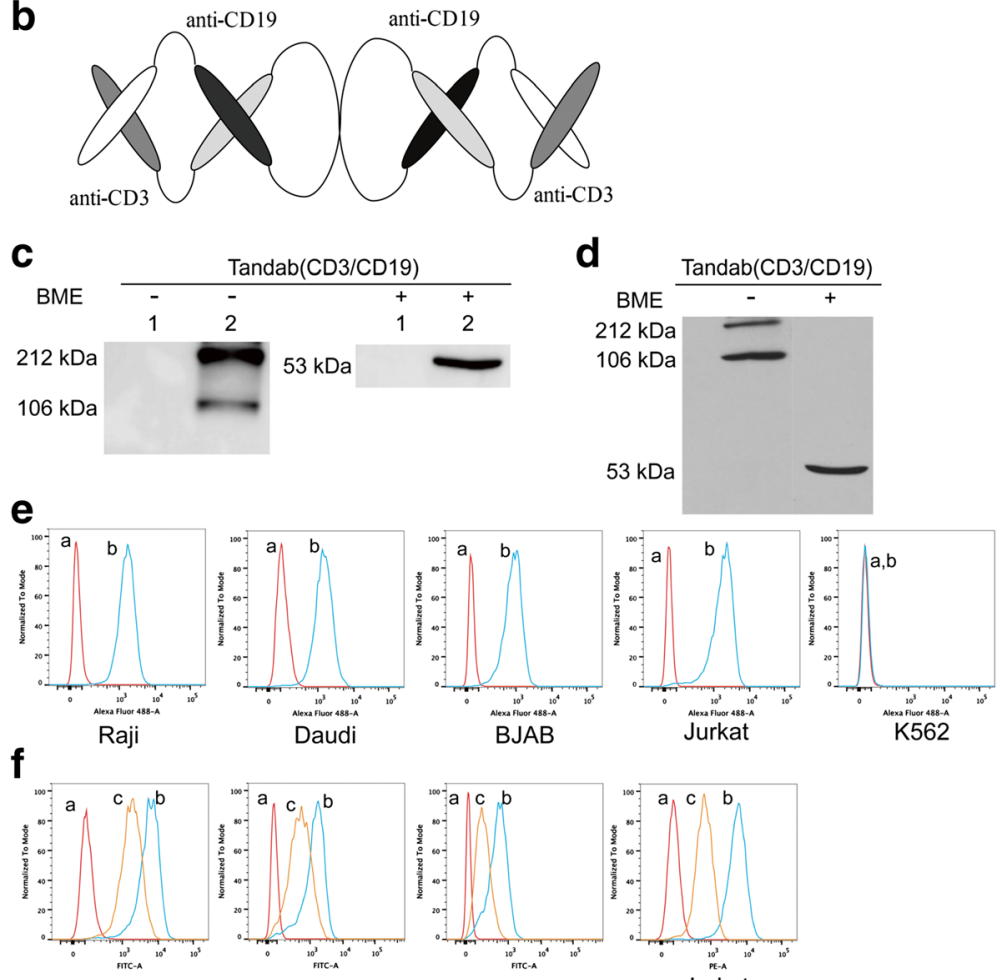

g
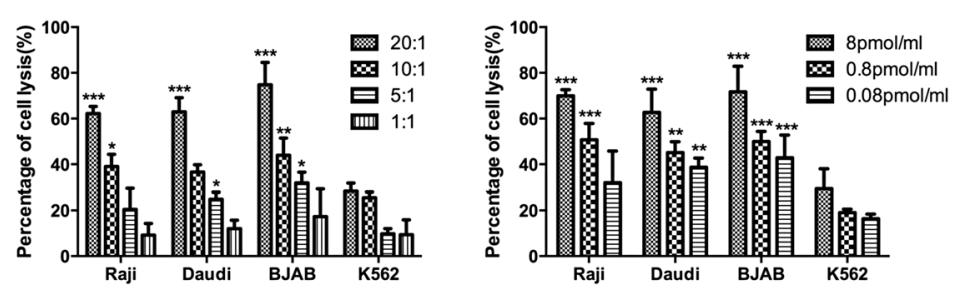

Fig. 1 Design, production, and biological function of bispecific tetravalent tandem diabody Tandab (CD3/CD19). a Schematic representation of lentiviral expression vector for Tandab (CD3/CD19). LTR, long terminal repeats; SV40, SV40 promoter; SP, signal peptide, a murine kappa lightchain leader peptide; G4S, Gly-Gly-Gly-Gly-Ser residues; His, hexa-histidine tag. b Molecular model of Tandab (CD3/CD19). Upon expression, two polypeptide gene products dimerize in a head-to-tail fashion. c Tandab (CD3/CD19) was determined by Western blot analysis. The supernatants from 293T cells after transfection with lentiviral expression vectors were harvested and assayed for Tandab (CD3/CD19) with or without $\beta$ mercaptoethanol (BME) using anti-His tag antibodies. Lane 1, 293T cells transfected with empty vector (negative control); lane 2, 293T cells transfected with vector expressing Tandab (CD3/CD19). $\mathbf{d}$ Western blot analysis of the purified Tandab (CD3/CD19). e Binding specificities of Tandab (CD3/CD19) to tumor cell lines. FACS analysis with the Tandab (CD3/CD19) on different CD19-positive B cell lines (Raji, Daudi, and BJAB), on CD3-positive Jurkat cells, and on the CD3- and CD19-negative K562 cells. $a$, negative controls with the secondary antibody anti-His-Alexa Fluor 488 alone; $b$, Tandab (CD3/CD19). f Competitive binding activity with FITC-conjugated HIT19a or PE-conjugated HIT3a. Cells were firstly incubated with Tandab (CD3/CD19) for $1 \mathrm{~h}$ at $4{ }^{\circ} \mathrm{C}$, then incubated with FITC-conjugated HIT19a or PE-conjugated HIT3a for 30 min at $4{ }^{\circ} \mathrm{C}$ before detection. a, Negative controls; b, FITC-conjugated HIT19a or PE-conjugated HIT3a alone; and c, Tandab (CD3/CD19) + FITC-conjugated HIT19a or PEconjugated HIT3a. Results are representative of three independent experiments. $\mathbf{g}$ Specific lysis of malignant target cell lines mediated by Tandab (CD3/CD19). Cytotoxicity of IL-2 pre-activated PBMCs induced by Tandab (CD3/CD19) with the same concentration (8 pmol/mL) in different effector to target (E:T) ratios ranging from 20:1 to 1:1 against CD19-positive B cell lines (Raji, Daudi, and BJAB) was detected by LDH release assay (left panel). Specific lysis of target cells by Tandab (CD3/CD19) with different concentrations at the same E:T ratio (20:1) was also determined (right panel). K562 cells were served as negative controls. ${ }^{*} P<0.05 ;{ }^{* *} P<0.01 ;{ }^{* * *} P<0.001$ compared with the corresponding K562 group. Data shown are the mean \pm SD of three independent experiments 
lysis of CD19-positive Raji cells, as well as the Daudi and BJAB cells (Fig. 1g). Furthermore, target cells were also lysed efficiently at the same E:T ratio (20:1) with different concentrations of Tandab (CD3/CD19) (Fig. 1g). Lysis of target cells induced by Tandab (CD3/CD19) proceeded in a dose-dependent manner, in which increasing the ratio of E:T or the concentration of Tandab (CD3/CD19) resulted in enhanced cytotoxicity. These results are in accordance with our earlier report on diabody of antiCD19/antiCD3 [23].

\section{MSCs can be successfully transduced to release Tandab (CD3/CD19)}

MSCs were isolated from human umbilical cord and identified according to previous reports [24, 25]. Then, MSCs were transduced with lentivirus encoding Tandab (CD3/CD19) with copGFP (MSC-Tandab) or alone copGFP (MSC-EV). The morphology of these cells and their transduction efficiency (indicated by copGFP) are shown in Fig. 2a. The transduction efficiency was also assessed by FACS analysis, which indicated that more than $73 \%$ of the MSCs were successfully transduced (Fig. 2b). And no significant alterations in cell survival were observed among MSC-Tandab, MSC-EV, and widetype MSCs (Fig. 2c). Western blot results demonstrated that Tandab (CD3/CD19) was highly expressed in MSCTandab but not in MSC-EV (Fig. 2d). Additionally, the Tandab (CD3/CD19) released in the supernatants in culture of MSC-Tandab was detected by ELISA. The level of secreted Tandab (CD3/CD19) achieved a peak at day $9(8247.2 \pm 796.7 \mathrm{pg} / \mathrm{mL})$ and was detectable even at day 15 after transduction (Fig. 2e). And there was no difference on surface markers between MSCs and transduced MSCs (Additional file 2: Fig. S1A). In addition, genemodified MSCs maintained their ability of tri-lineages differentiation (Additional file 2: Fig. S1B and C).

\section{Homing property of MSCs to B cell lymphoma in vitro and in vivo}

MSCs and gene-modified MSCs were previously proved to have a homing predisposition to tumor cells in vitro and to the tumor site in models of hepatocarcinoma with HepG2 cells or lymphoma with BJAB cells $[12,13,25]$. To investigate the migration capacity of transduced MSCs to another B cell lymphoma cell line (Raji cells) in this study, migration assays in vitro using transwell plates were

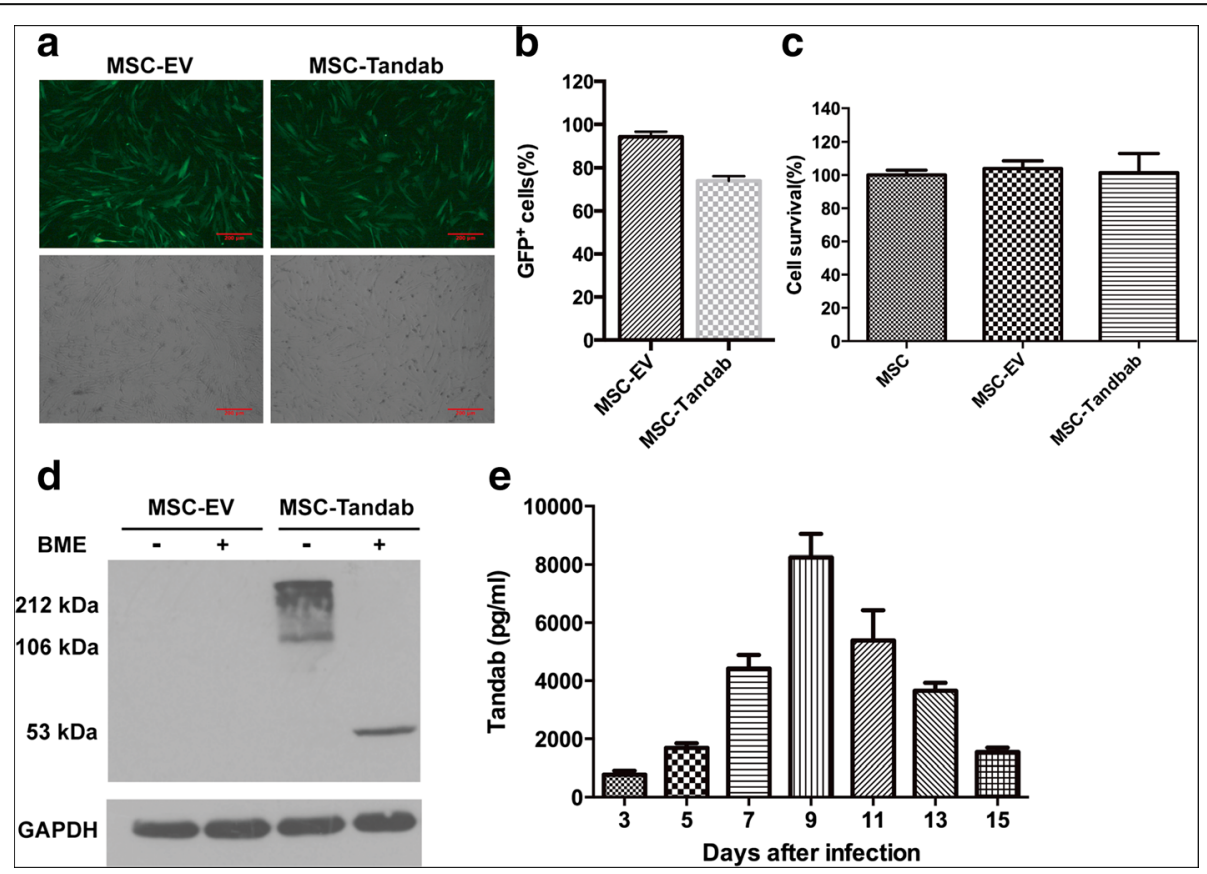

Fig. 2 Constitutive expression of Tandab (CD3/CD19) in MSCs. MSCs were transduced with lentivirus coding Tandab (CD3/CD19) at 8 MOI for $12 \mathrm{~h}$. Then, supernatants were removed, and fresh medium culture was added. a The representative images depicted the infection efficiency of MSCs with lentivirus. Forty-eight hours after infection, MSCs carrying copGFP were observed under fluorescent field (upper panel) and bright field (lower panel), scale bar = $\mu \mathrm{m}$. MSC-Tandab, MSCs transduced with lentivirus coding Tandab (CD3/CD19); MSC-EV, MSCs transduced with empty lentivirus. b FACS analysis of percentages of copGFP-positive MSCs. c Cell survival of MSCs transduced with or without lentivirus were detected by the MTT assay. $\mathbf{d}$ Western blot analysis was employed to determine the protein expression of Tandab (CD3/CD19) in MSCs with anti-His tag antibodies after 5 days of transduction. GAPDH, served as a loading control; BME, $\beta$-mercaptoethanol. e Transduced MSCs secreted Tandab (CD3/ CD19) constantly. MSC-Tandab and MSC-EV were cultured in a 24-well plate $\left(4 \times 10^{4} /\right.$ well). And the level of Tandab (CD3/CD19) released into culture was measured by ELISA in the indicated time. Data shown are the mean \pm SD of the three repeated experiments 
performed. Culture medium (CM) without Raji cells was served as a negative control of chemotaxis, and unmodified MSCs were used as a positive control of migrating cells. We confirmed that the transduced MSCs migrated towards Raji cultures in a similar pattern as unmodified MSCs (Fig. 3a, b). These results indicated that human B cell lymphoma Raji cells were able to stimulate the migration of MSCs and the migration capacity of MSCs was not affected by infection of lentivirus.

In order to verify the homing ability of MSCs in vivo, we designed a lentiviral vector (pLeniR-Luc) harboring a firefly luciferase reporter gene (Fig. 3c). MSCs labeled with luciferase (MSC-Luc) were analyzed at first ex vivo using bioluminescence imaging (BLI) system (Fig. 3d), which suggested that luciferase reporter gene could be used to quantify transplanted MSCs in small living animals. Then, MSC-Luc $\left(1 \times 10^{6}\right.$ cells per mouse $)$ was injected intravenously into $\mathrm{BALB} / \mathrm{c}$ nude mice with established subcutaneously tumor with Raji cells. BLI revealed that MSCs migrated and selectively accumulated at the tumor site at $24 \mathrm{~h}$ after injection although part of MSCs was hijacked in the lungs, in which the signal decreased as time went on (Fig. 3e). The strongest signal in the tumor site was observed on day 2, and it was out of detectable on day 5 (Fig. 3e). During the monitoring process, MSCs were not traced in other tissues.

\section{MSC-Tandab exhibits cytotoxicity against CD19-positive Raji cells}

To confirm the bioactivity of secreted Tandab (CD3/ CD19) from MSC-Tandab, the co-culture system using transwell plates with $0.4-\mu \mathrm{m}$-pore membrane (cells could not pass through) was designed. MSC-secreting Tandab (CD3/CD19), concentration of which could reach

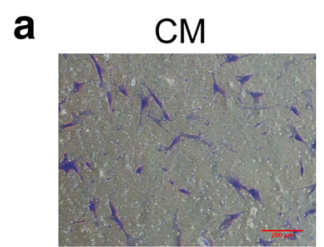

b

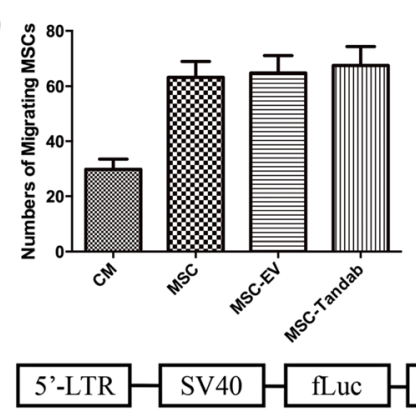

e

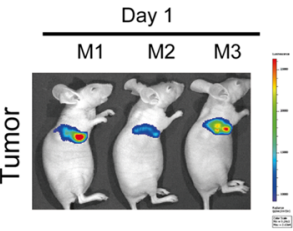

Day 1

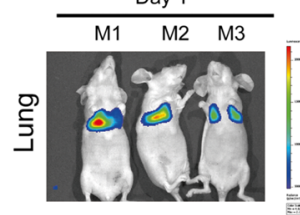

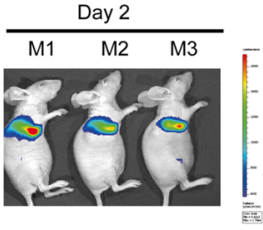

Day 2

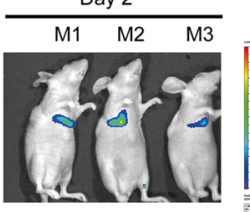

\section{MSC-EV}

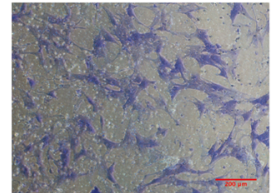

MSC-Tandab

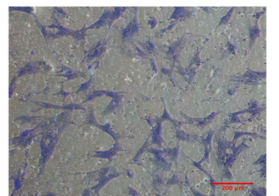

d

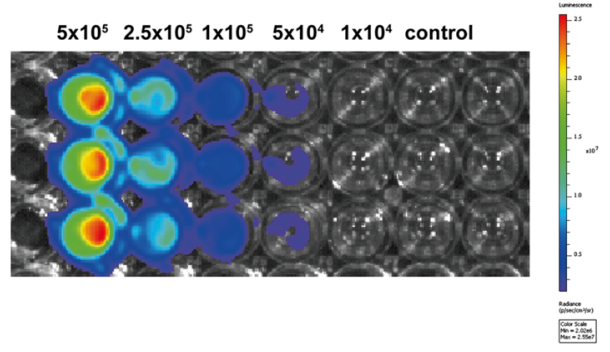

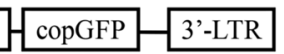
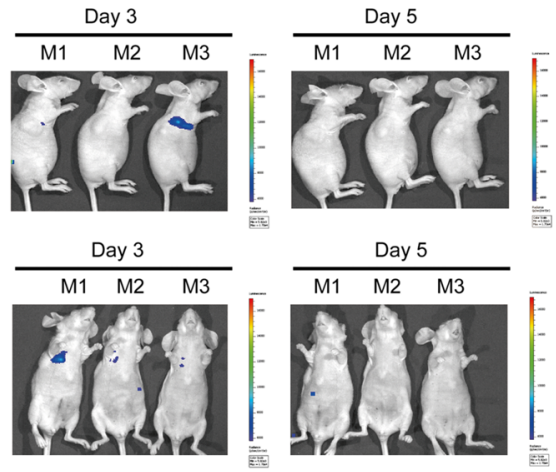

Fig. 3 Migration capacity of MSCs to B cell lymphoma in vitro and in vivo. a Representative photographs showed the migrated MSCs stained with crystal violet in vitro migration assays. Culture medium (CM), served as a negative control. Scale bar $=200 \mu \mathrm{m}$. $\mathbf{b}$ The numbers of migrated MSCs in three independent assays were expressed as mean \pm SD. c Schematic representation of lentiviral expression vector for firefly luciferase (Luc). d MSC-Luc expressed Luc constitutively in vitro. e Tropism of MSCs to tumor site. MSC-Luc was intravenously injected into tumor-bearing mice. The mice were anesthetized in the indicated time and received intraperitoneal injection of D-luciferin at a dose of $150 \mu \mathrm{g}$ of D-luciferin per gram of body weight. Ten minutes later, the BLI for luciferase activity was detected by Xenogen in vivo imaging system. M1, M2, M3: represented three mice 
$(0.1516 \pm 0.0283) \mathrm{pmol} / \mathrm{mL}$, in the lower chamber could pass through the membrane followed by triggering reactions between PBMCs and Raji cells $(\mathrm{E}: \mathrm{T}=10: 1)$. After incubation for $24 \mathrm{~h}$, obviously lysis $(60.6 \pm 5.7 \%)$ of Raji cells was detected by flow cytometry (Fig. 4a). The expression levels of the early $\mathrm{T}$ cell activation marker CD69 and of the late activation marker CD25 on CD3posivitve cells were also assessed (Fig. 4b). Since the increase of cytokines concentration is the response of $\mathrm{T}$ cells activation and cytotoxicity, classic cytokines including IL-2, IFN- $\gamma$, and TNF- $\alpha$ were measured as an example to evaluate the activation efficacy of $\mathrm{T}$ cells in the co-culture system. The concentrations of IL- 2, IFN- $\gamma$, and TNF- $\alpha$ were $(708.27 \pm 36.16) \mathrm{pg} / \mathrm{mL}$, $(30.31 \pm 2.69) \mathrm{ng} / \mathrm{mL}$, and $(60.66 \pm 8.21) \mathrm{pg} / \mathrm{mL}$ in the supernatant, respectively, all of which were significantly higher than that of the control groups $(P<0.001)$ (Fig. 4c-e). These data suggest that MSC-Tandab can trigger cytotoxicity to CD19-positive tumor cells in the presence of effector cells.

\section{The cytotoxicity induced by MSC-secreting Tandab (CD3/CD19) can be enhanced by IDO pathway inhibitor D-1MT}

Although we have demonstrated that CD19-positive tumor cells could be killed efficiently by $\mathrm{T}$ cells in the co-culture system, the presence of MSCs should be in consideration because of their great immune-modulating capacity. Some earlier reports have indicated that IDO plays a pivotal role in MSC-mediated immunosuppression [17, 31, 32] because IDO expression by human MSCs occurs only after exposure to inflammatory cytokines such as IFN- $\gamma[31,33]$, which produced massively by $\mathrm{T}$ cells during the co-culture assays. For this reason, an IDO pathway inhibitor D-1MT was employed in our study to overcome the immunosuppression induced by MSCs. We firstly verified the inducible expression of IDO in MSCs with stimulation of exogenous IFN- $\gamma$. After stimulation with IFN- $\gamma(20 \mathrm{ng} / \mathrm{mL})$ for $48 \mathrm{~h}$, the expression of IDO were detected at the mRNA level and protein level (Fig. 5a, b). Furthermore, the enzymatic activity of IDO (indicated by concentration of kynurenine, a metabolism of tryptophan) was measured (Fig. 5c). We next determined whether D-1MT had any cytotoxicity on MSCs, Raji cells, or PBMCs. Results from the cell proliferation assays suggested that no obvious influences were caused by D-1MT on those cells (Fig. 5d). Based on these results, we then performed a co-culture killing experiment again in the same conditions described above in the absence or presence of D-1MT $(1 \mathrm{mM})$ for 24, 48, and $72 \mathrm{~h}$. As shown in Fig. 5e, the number of residual Raji cells decreased significantly at 48 and $72 \mathrm{~h}$ $(P<0.05)$. However, there was no significant difference at the first $24 \mathrm{~h}$.
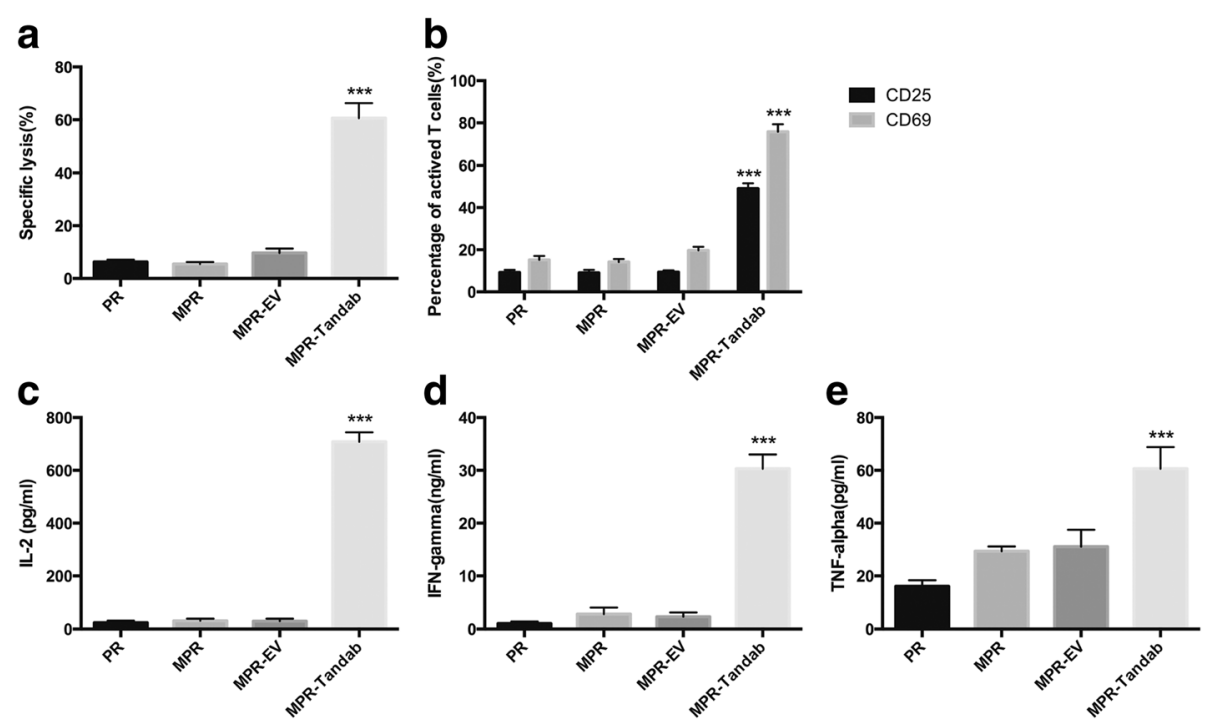

Fig. 4 Cytotoxicity of T cells to Raji cells mediated by Tandab (CD3/CD19) secreted from MSCs. In order to evaluate the function of MSCs-secreting Tandab (CD3/CD19), a co-culture system using transwell plates with $0.4-\mu \mathrm{m}$-pore membrane was established. MSCs were plated into 6-well plates with a density of $1 \times 10^{5}$ cells per well after transduced with lentivirus. Seventy-two hours later, Raji cells were labeled with calcein-AM (5 $\mu \mathrm{M})$. Then, PBMCs and labeled Raji cells (E:T=10:1) were added to the equilibrated inserts. After co-cultured for $24 \mathrm{~h}$, cells in the inserts were harvested to be detected by FACS. a The specific lysis of Raji cells. b Activation surface markers CD69 and CD25 of T cells. c-e Cytokines including IL-2, IFN- $\gamma$, and TNF- $a$ in the supernatant were measured using ELISA kits. PR, PBMC + Raji; MPR, MSC + PBMC+Raji; MPR-EV, MSC-EV + PBMC+Raji; MPR-Tandab, MSC-Tandab + PBMC + Raji. ***P< 0.001 compared with PR group. Data shown are the mean \pm SD of the three repeated experiments 

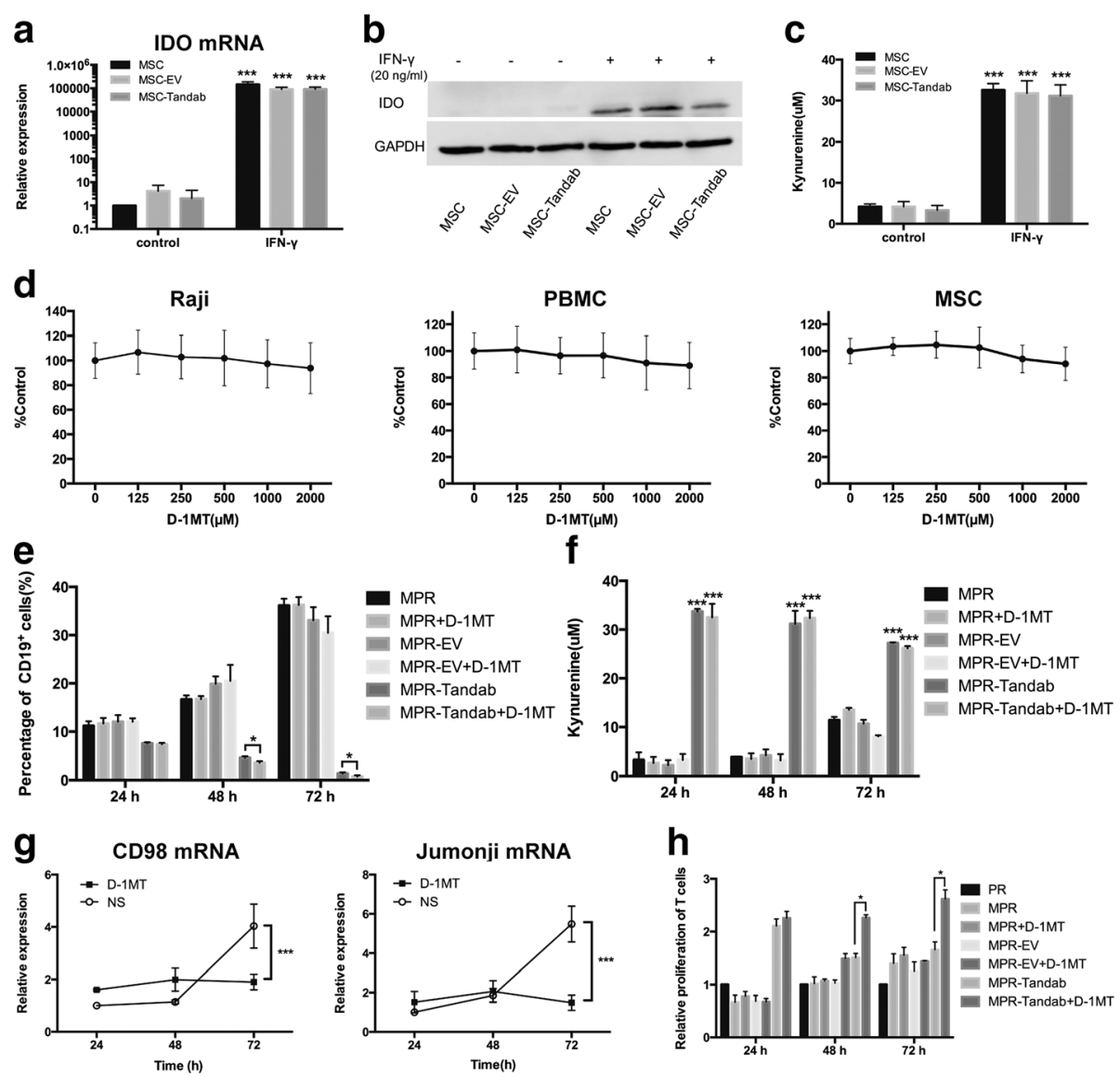

Fig. 5 IDO pathway inhibitor D-1MT enhances the cytotoxicity induced by MSC-secreting Tandab (CD3/CD19) in vitro. a IDO mRNA expression in MSCs stimulated with IFN- $\gamma$. MSCs were cultured in the absence or presence of exogenous IFN- $\gamma(20 \mathrm{ng} / \mathrm{mL})$. Cells were harvested after 2 days in culture, total mRNA was extracted, and IDO message was assayed by real-time PCR. b IDO protein was determined by Western blot analysis. MSCs were harvested after cultured for $48 \mathrm{~h}$, as in $\mathbf{a}$, and total cell lysates were assayed for IDO protein. Results are representative of three independent experiments. c To determine enzyme activity of IDO, MSCs were cultured with or without IFN- $(20 \mathrm{ng} / \mathrm{mL})$ stimulation for $48 \mathrm{~h}$. IDO enzyme activity was evaluated by spectrophotometric detection of the tryptophan metabolite, kynurenine, a product of IDO catabolism. $\mathbf{d}$ Influence of D-1MT on the proliferation of cells (Raji, PBMCs and MSCs). Cells were seeded in 96-well plates and treated with different concentrations of D-1MT (0-2000 $\mu \mathrm{M})$ for $72 \mathrm{~h}$. Cell proliferation was determined by MTT assay, and the $y$ axis represents cell proliferation as a percent of the control. e D-1MT promotes the specific lysis of Raji cells induced by MSC-secreting Tandab (CD3/CD19). MSCs, Raji cells, and PBMCs were co-cultured as mentioned above with or without D-1MT (1 mM) for 24, 48, and $72 \mathrm{~h}$. The residual Raji cells were detected by FACS with FITCconjugated anti-CD19 antibodies. D-1MT, D-1-methyl-tryptophan. $\mathbf{f}$ Determination of kynurenine in the supernatant from the co-culture system. g Analysis of anergy-associated genes expression of CD98 (left panel) and Jumonji (right panel). The cells in the inserts were harvested in the indicated time. Total mRNA was extracted, and the messages of CD98 and Jumonji were assayed by real-time PCR. NS, normal saline. $\mathbf{h}$ Relative proliferation of T cells. The cells were harvested in the indicated time. Proliferation of T cells was detected using BrdU flow kit. The proliferation of T cells in PR group (without MSCS) was served as a reference. ${ }^{*} P<0.05$ compared with the control group; ${ }^{* * *} P<0.001$ compared with the control group. Data shown are the mean \pm SD of the three repeated experiments

To further investigate the mechanism of D-1MT exerted in the increasing cytotoxicity, we firstly pointed to the level of kynurenine which had no changes from 24 to $72 \mathrm{~h}$ (Fig. 5f). Previously, reports show that D-1MT can act as a mimetic of tryptophan in the sufficiency pathway, thereby functionally reversing the effects of IDO on formation of $T$ cell anergy controlled by PKC- $\theta$ [22]. We therefore examined the effect of D-1MT on the expression of anergyassociated genes in T cells, including CD98 and Jumonji [34]. Both of the expression of CD98 and Jumonji in T cells from the co-culture system were up-regulated at $72 \mathrm{~h}$, but this effect was reversed efficiently by D-1MT (Fig. 5g). Interestingly, the proliferation of $\mathrm{T}$ cells in the co-culture system was also partly restored by D-1MT at 48 and 72 h, respectively (Fig. 5h).

\section{Antitumor potential of MSC-Tandab in combination with D-1MT against Raji xenograft tumors}

We further investigated the antitumor potential of MSCs engineered with Tandab (CD3/CD19) in combination 
with D-1MT in BALB/c nude mice subcutaneously implanted with Raji cells. Firstly, to ascertain the safety of this therapeutic schedule, tumor-bearing mice were treated with MSC-Tandab, PBMCs, and D-1MT. Results from pathological reports indicated that this treatment did not cause organ damages, such as the lung, liver, spleen, and kidney (Fig. 6a). Then, we begun the formal therapeutic experiments. When the tumor size reached $100-200 \mathrm{~mm}^{3}$, the tumor-bearing mice were grouped and treatments were started according to the scheme shown in Fig. 6b. The animals were sacrificed on day 21, and tumor tissues were dissected and weighed for analysis. Interestingly, compared with the mice in the control group (treated with MSC-EV + PBMC), the mice treated with MSC-Tandab and PBMCs exhibited no evident tumor regression (Fig. 6c). However, the tumor weight of the mice treated with MSC-Tandab and PBMCs combined with D-1MT decreased 61.2\% (Fig. 6c). In addition, no obvious changes in the body weight of the mice were observed during the treatment (Fig. 6d).

\section{Discussion}

Application of MSCs to serve as vehicles for delivering TandAb to tumor has not been reported so far. In this study, we investigated the therapeutic effects of genemodified MSCs with Tandab (CD3/CD19) for B cell lymphoma. The hypothesis is that engineered MSCs which injected intravenously into the tumor-bearing mice would specifically migrate to tumor site and secrete Tandab (CD3/CD19) which recruits $\mathrm{T}$ cells to exhibit a potent antitumor immunity in combination with an IDO pathway inhibitor D-1MT. And results presented here support the feasibility of this strategy.

Currently, two approaches have been developed to harness $\mathrm{T}$ cells for killing tumor cells. The first approach utilizes gene-modified $\mathrm{T}$ cells with an engineered chimeric antigen receptor (CAR) combining a desired antigen-recognition fragment of a monoclonal antibody against $\mathrm{T}$ cell activation domains from the $\mathrm{T}$ cell receptor complex, such as the $\zeta$ chain, with co-stimulatory molecules, from CD28, 4-1BB, or OX40 [35]. The other

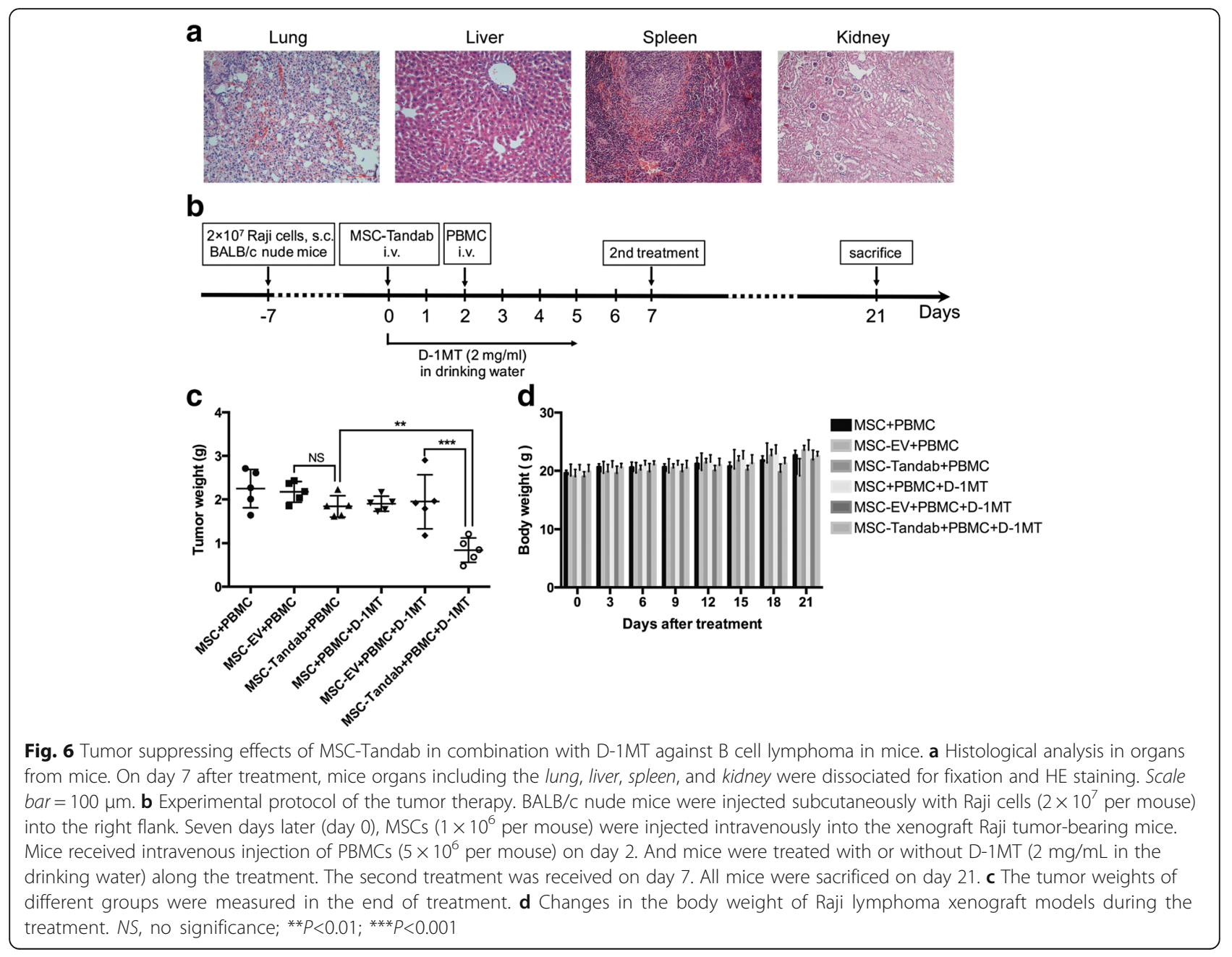


approach depends on $\mathrm{T}$ cell recruitment via bispecific antibodies (bsAbs) [36] which bind one arm to T cell activation domain and bind the other arm to a tumorassociated antigen on the target cell. We previously constructed an anti-CD3 $\times$ anti-CD19 bispecific antibody, which could efficiently redirect $\mathrm{T}$ cells to lysis both $\mathrm{B}$ cell lymphoma cell lines and patient-derived B-ALL cells $[23,37]$. In this study, we successfully constructed a new tandem diabody with two binding sites for CD3 and two for CD19 using the DNA sequences from our antiCD3 $\times$ anti-CD19 bispecific antibody. The relative amount of tandem diabodies proved to be dependent on the length of the linker in the middle of the chain [7]. It was reported that the middle linker should not be too short or too long to force the four domains to interact with complementary ones of another molecule with the formation of an eight-domain Tandab [7]. We therefore chose a linker with 15 amino acid residues in our construct. In addition, our earlier data have suggested that the introduced disulfide covalent bond between $\mathrm{V}_{\mathrm{H}} 3$ and $\mathrm{V}_{\mathrm{L}} 3$ at rational positions could enhance the stability of fusion proteins $[23,38]$. Since the two peptides of Tand $\mathrm{Ab}$ are held together by non-covalent associations of the corresponding $\mathrm{V}_{\mathrm{H}}$ and $\mathrm{V}_{\mathrm{L}}$ domains, an extra disulfide bond can prevent the peptide diffusing away from the other one. Results from Western blot analysis suggested that nearly no monomers were detectable in the nonreducing condition. However, we also observed a band in $212 \mathrm{kDa}$ in the non-reducing condition (Figs. 1c, d and $2 \mathrm{~d})$, which should be dimers of Tandab (CD3/CD19) with intermolecular forces. And the amount of them decreased after purification (Fig. 1d). Furthermore, data from cellbinding assays and cytotoxicity assays in vitro indicated that our Tandab (CD3/CD19) could bind to both of CD3positive cells and CD19-positive cells and induce the specific lysis of CD19-positive cells.

MSCs hold great promise for clinical applications in the treatment of various diseases owing to their multilineage differentiation potential and immunosuppressive properties. Furthermore, the tumor tropism property of MSCs has led to the utilization of them as attractive delivery vehicles for a spectrum of antitumor agents against cancers [39-43]. Several previous investigations reported by our laboratory also support feasibility of this strategy $[12,13,25,44]$. In the present study, we engineered the human umbilical cord-derived MSCs through transduction by lentivirus to secrete Tandab (CD3/ CD19). We demonstrated that the MSC-Tandab could maintain their properties after lentiviral infection, including their surface markers, tri-lineage differentiation, proliferation, and the capacity to migrate towards tumor cells in vitro. Additionally, the tropism towards tumorbearing mice with Raji cells was confirmed by in vivo imaging system with luciferase-labeled MSCs (Fig. 3e).
These findings indicate that UC-MSCs can be used as ideal vehicles to deliver Tandab (CD3/CD19) to tumor.

Furthermore, to mimic the therapeutic process with MSC-Tandab in vivo, we designed a co-culture system using transwell plates mentioned above, in which Tandab (CD3/CD19) secreted from MSC-Tandab could migrate to the upper insert to trigger cytotoxicity of $\mathrm{T}$ cells against CD19-positive cells. Results from the specific lysis of Raji cells and released cytokines confirmed our initial hypothesis. However, it has been reported that various factors are believed to be involved in the mechanisms of MSC-mediated immunosuppression, including inducible nitric oxide synthase (iNOS), IDO, tumor necrosis factor-inducible gene 6 (TSG6), CC-chemokine ligand 2 (CCL2), IL-10, and prostaglandin E2 (PGE2) [45]. Although the immune-regulatory effects of MSCs do not occur spontaneously, it should be taken into account in this study because of the released cytokines, such as IFN- $\gamma$. The expression of IDO in MSCs induced by IFN $-\gamma$ might contribute to the immunosuppressive microenvironment owing to tryptophan depletion and accumulation of its metabolites. Additionally, MSCTandab shows basal increased levels of kynurenine (Fig. 5f), which is associated to an enhanced immunosuppression ability. This aspect indicates that we should pay attention to genetic modification of MSCs since they could be responsible of an augmented pro-tumoral effect. Therefore, we employed D-1MT, an IDO pathway inhibitor which differed from direct enzymatic inhibitors of IDO, to overcome the inhibition effect of IDO. Data from the co-culture killing experiments showed that D1MT could increase the ratio of lytic cells at a certain degree. No significant changes on the level of kynurenine were observed, which suggested that D-1MT could not inhibit the enzymatic activity of IDO directly. Then, we confirmed that the expression of the $\mathrm{T}$ cell anergyassociated genes CD98 and Jumonji were decreased in the presence of D-1MT. And the proliferation capacity of $\mathrm{T}$ cells was also increased by D-1MT. Furthermore, the antitumor growth effect of MSC-Tandab in vivo was enhanced significantly in combination with D-MT. Although IDO is known to be overexpressed in several human cancers, including prostate, breast, brain, and hematologic malignancies [46], it is not detectable in Raji cells even with the stimulation of IFN- $\gamma$ [47]. While a study about IDO expression by circulating leukemic cells from 5 B-CLL patients showed that IDO could not be detected in the PBMCs of any of them, but in four of the five, IDO was dramatically up-regulated when cells were cultured with IFN- $\gamma$ for $24 \mathrm{~h}$ [47]. This may be one of the reasons for drug resistance during the treatment. Thus, our treatment with MSC-Tandab in combination with IDO pathway inhibitor D-1MT indicates a new treatment strategy for refractory and relapse B cell malignancies. 


\section{Conclusions}

We have demonstrated in this study that human UCMSCs can be acted as cell-based delivery vehicles for the treatment of B cell lymphoma. Tandab (CD3/CD19) secreted from UC-MSCs effectively redirected T cells to inhibit the growth of Raji lymphoma in mouse models in combination with D-1MT. Our findings indicate that the use of UC-MSCs as vehicles for engineered-antibodies combined with immunoregulatory agents represents a potential clinical application of gene therapy for cancers.

\section{Additional files}

Additional file 1: Supplementary methods and materials. (DOCX $100 \mathrm{~kb}$ )

Additional file 2: Figure S1. Identification of MSCs and transduced MSCs. A. Phenotypic analysis of MSCs and transduced MSCs. Flow cytometric analysis showed that MSCs, MSC-EV, and MSC-Tandab expressed CD73, CD90, and CD105 but not CD14, CD19, CD34, CD45, and HLA-DR. B. Representative images showing the in vitro differentiation of MSCs and transduced MSCs into adipogenic, osteogenic, and chondrogenic lineages, respectively. Scale bar $=200 \mu \mathrm{m}$. C. Relative quantification of gene expression after tri-lineage differentiation in MSCs and transduced MSCs. The mRNA levels were normalized using the expression of the reference gene (GAPDH) and compared with the MSCs group. Data shown are the mean \pm SD of three repeated experiments. (TIF $9594 \mathrm{~kb}$ )

\section{Abbreviations}

AHR: Aryl hydrocarbon receptor; B-ALL: B-precursor acute lymphoblastic leukemia; BiTE: Bispecific T cell engager; BLI: Bioluminescent imaging; BNHL: B cell non-Hodgkin's lymphoma; BrdU: Bromodeoxyuridine; bsAbs: Bispecific antibodies; CAR: Chimeric antigen receptor; CM: Culture medium; D-1MT: D-1-methly-tryptophan; ELISA: Enzyme-linked immune sorbent assay; EV: Empty vector; FDA: Food and Drug Administration; GAS: $\gamma$ activating sequences; IDO: Indoleamine 2,3-dioxygenase; ISRE: Interferon stimulatory response elements; Luc: Luciferase; MOI: Multiplicity of infection; MRD: Minimal residue diseases; MSCs: Mesenchymal stromal cells; PBMCs: Peripheral blood mononuclear cells; PBS: Phosphate-buffered saline solution; PCR: Polymerase chain reaction; scFv: Single-chain variable fragment; TandAb: Tandem diabody; UC: Umbilical cord; WJ: Wharton's jelly

\section{Acknowledgements}

Not applicable.

\section{Funding}

This work was supported by the National Natural Science Foundation of China (Grant Nos. 30971291, 81400176, 81572993), National Science and Technology Major Project (Grant No. 2012zx09102301-015), and CAMS Initiative for Innovative Medicine (Grant Nos. 2016-I2M-1-007, 2016-I2M-3-013).

\section{Availability of data and materials}

The dataset supporting the conclusions of this article is included within the article.

\section{Authors' contributions}

$X Z$ constructed the lentiviral expression vector and performed the cytotoxicity assay in vitro and therapeutic experiments in vivo. YY and LZ participated in the tri-lineage differentiation assays. $Y Y$ and $Y L$ contributed to animal experiments. QZ isolated PBMCs from peripheral blood and MSCs from umbilical cord. DF finished the binding assay of Tandab (CD3/CD19) and was responsible for the culture of cells in this study. YiZ, YaZ, and ZY participated in the manuscript preparation and revisions. DX was the corresponding author and contributed to the design of this subject. All authors read and approved the final manuscript.

\section{Competing interests}

The authors declare that they have no competing interests.

\section{Consent for publication}

Not applicable.

\section{Ethics approval}

All animal studies were performed in accordance with the guidelines under the Animal Ethics Committee of the Institute of Hematology and Hospital of Blood Diseases, Chinese Academy of Medical Sciences and Peking Union Medical College.

Received: 28 September 2016 Accepted: 11 January 2017

Published online: 23 February 2017

\section{References}

1. Grillo-Lopez AJ, White CA, Dallaire BK, Varns CL, Shen CD, Wei A, et al. Rituximab: the first monoclonal antibody approved for the treatment of lymphoma. Curr Pharm Biotechnol. 2000;1(1):1-9.

2. Hato T, Yamanouchi J, Tamura T, Hojo N, Niiya Y, Kohno M, et al. Existence of leukemic clones resistant to both imatinib mesylate and rituximab before drug therapies in a patient with Philadelphia chromosome-positive acute lymphocytic leukemia. Int J Hematol. 2004;80:62-6.

3. Baeuerle PA, Kufer P, Lutterbuse R. Bispecific antibodies for polyclonal T-cell engagement. Curr Opin Mol Ther. 2003:5:413-9.

4. Kaplan JB, Grischenko M, Giles FJ. Blinatumomab for the treatment of acute lymphoblastic leukemia. Invest New Drugs. 2015;33:1271-9.

5. Viardot A, Goebeler M-E, Hess G, Neumann S, Pfreundschuh M, Adrian N, et al. Phase 2 study of the bispecific T-cell engager (BiTE) antibody blinatumomab in relapsed/refractory diffuse large B-cell lymphoma. Blood. 2016;127:1410-6.

6. Le Jeune $C$, Thomas $X$. Potential for bispecific T-cell engagers: role of blinatumomab in acute lymphoblastic leukemia. Drug Des Devel Ther. 2016;10:757-65.

7. Kipriyanov SM, Moldenhauer G, Schuhmacher J, Cochlovius B, Von der Lieth C-W, Matys ER, et al. Bispecific tandem diabody for tumor therapy with improved antigen binding and pharmacokinetics. J Mol Biol. 1999;293:41-56.

8. Reusch U, Duell J, Ellwanger K, Herbrecht C, Knackmuss SH, Fucek I, et al. A tetravalent bispecific TandAb (CD19/CD3), AFM11, efficiently recruits T cells for the potent lysis of CD19 ${ }^{+}$tumor cells. mAbs. 2015;7:584-604.

9. Brüggemann M, Raff T, Flohr T, Gökbuget N, Nakao M, Droese J, et al. Clinical significance of minimal residual disease quantification in adult patients with standard-risk acute lymphoblastic leukemia. Blood. 2006;107:1116.

10. Studeny M, Marini FC, Dembinski JL, Zompetta C, Cabreira-Hansen M, Bekele BN, et al. Mesenchymal stem cells: potential precursors for tumor stroma and targeted-delivery vehicles for anticancer agents. JNCI J Natl Cancer Inst. 2004;96:1593-603.

11. Mohr A, Albarenque SM, Deedigan L, Yu R, Reidy M, Fulda S, et al. Targeting of XIAP combined with systemic mesenchymal stem cell-mediated delivery of sTRAIL ligand inhibits metastatic growth of pancreatic carcinoma cells. Stem Cells. 2010;28:2109-20.

12. Yan C, Li S, Li Z, Peng H, Yuan $X$, Jiang L, et al. Human umbilical cord mesenchymal stem cells as vehicles of CD20-specific TRAIL fusion protein delivery: a double-target therapy against non-Hodgkin's lymphoma. Mol Pharm. 2013;10:142-51.

13. Yan C, Yang M, Li Z, Li S, Hu X, Fan D, et al. Suppression of orthotopically implanted hepatocarcinoma in mice by umbilical cord-derived mesenchymal stem cells with sTRAlL gene expression driven by AFP promoter. Biomaterials. 2014;35:3035-43

14. Kim D-W, Staples M, Shinozuka K, Pantcheva P, Kang S-D, Borlongan C. Wharton's jelly-derived mesenchymal stem cells: phenotypic characterization and optimizing their therapeutic potential for clinical applications. Int J Mol Sci. 2013;14:11692-712.

15. Ribeiro A, Laranjeira P, Mendes S, Velada I, Leite C, Andrade P, et al. Mesenchymal stem cells from umbilical cord matrix, adipose tissue and bone marrow exhibit different capability to suppress peripheral blood B, natural killer and T cells. Stem Cell Res Ther. 2013;4:125.

16. Platten M, Wick W, Van den Eynde BJ. Tryptophan catabolism in cancer: beyond IDO and tryptophan depletion. Cancer Res. 2012;72:5435.

17. Ling W, Zhang J, Yuan Z, Ren G, Zhang L, Chen X, et al. Mesenchymal stem cells use IDO to regulate immunity in tumor microenvironment. Cancer Res. 2014;74:1576-87. 
18. Friberg $M$, Jennings $R$, Alsarraj $M$, Dessureault $S$, Cantor A, Extermann $M$, et al. Indoleamine 2,3-dioxygenase contributes to tumor cell evasion of $\mathrm{T}$ cell-mediated rejection. Int J Cancer. 2002;101:151-5.

19. Frumento G, Rotondo R, Tonetti M, Damonte G, Benatti U, Ferrara GB. Tryptophan-derived catabolites are responsible for inhibition of $\mathrm{T}$ and natural killer cell proliferation induced by indoleamine 2,3-dioxygenase. J Exp Med. 2002;196:459-68.

20. Braun D, Longman RS, Albert ML. A two-step induction of indoleamine 2,3 dioxygenase (IDO) activity during dendritic-cell maturation. Blood. 2005:106:2375-81

21. Carlin JM, Borden EC, Sondel PM, Byrne Gl. Interferon-induced indoleamine 2,3-dioxygenase activity in human mononuclear phagocytes. J Leukoc Biol. 1989:45:29-34

22. Metz R, Rust S, Duhadaway JB, Mautino MR, Munn DH, Vahanian NN, et al. IDO inhibits a tryptophan sufficiency signal that stimulates mTOR: a novel IDO effector pathway targeted by D-1-methyl-tryptophan. Oncolmmunology. 2012:1:1460-8.

23. Wei L, Dongmei F, Ming Y, Ruizan S, Yan Y, Linlin J, et al. Disulfide-stabilized diabody antiCD19/antiCD3 exceeds its parental antibody in tumor-targeting activity. Cell Oncol. 2012;35:423-34.

24. Ma L, Feng $X$, Cui B, Law $F$, Jiang $X$, Yang $L$, et al. Human umbilical cord Wharton's Jelly-derived mesenchymal stem cells differentiation into nervelike cells. Chin Med J. 2005;118:1987-93.

25. Li Z, Ye Z, Zhang X, Zhang Q, Fan D, Zhang Y, et al. E1A-engineered human umbilical cord mesenchymal stem cells as carriers and amplifiers for adenovirus suppress hepatocarcinoma in mice. Oncotarget. 2016:7(32):51815-28.

26. Thakur A, Zaman A, Hummel J, Jones K, Hortelano G. Single-colour flow cytometric assay to determine NK cell-mediated cytotoxicity and viability against non-adherent human tumor cells. Biotechnol Lett. 2012;34:447-53.

27. Holliger P, Prospero T, Winter G. Diabodies- small bivalent and bispecific antibody fragments. Proc Natl Acad Sci U S A. 1993;90(14):6444-8.

28. Chen S, Rao Q, Wang J, Wang M. Construction and expression of single chain variable fragments (SCFv) against human CD19 antigen. Chin J Biotechnol. 2005;21:686-91.

29. Shen D, Yang X, Yang C, Tang M, Zhang J, Bai J, et al. A high affinity CD3 monoclonal antibody HIT3a. I production and identification. Zhong Guo Yi Xue Ke Xue Yuan Xue Bao. 1993;15:157-62.

30. An N, Tao Z, Li S, Xing H, Tang K, Tian Z, et al. Construction of a new antiCD19 chimeric antigen receptor and the anti-leukemia function study of the transduced T cells. Oncotarget. 2016;7(9):10638-49.

31. Meisel R. Human bone marrow stromal cells inhibit allogeneic T-cell responses by indoleamine 2,3-dioxygenase-mediated tryptophan degradation. Blood. 2004;103:4619-21.

32. Hong J, Hueckelhoven A, Wang L, Schmitt A, Wuchter P, Tabarkiewicz J, et al. Indoleamine 2,3-dioxygenase mediates inhibition of virus-specific CD8+ T cell proliferation by human mesenchymal stromal cells. Cytotherapy. 2016;18:621-9.

33. Abumaree M, Al Jumah M, Pace RA, Kalionis B. Immunosuppressive properties of mesenchymal stem cells. Stem Cell Rev Rep. 2012;8:375-92.

34. Li T, Wong Wai VK, Yi XQ, Wong YF, Zhou H, Liu L. Matrine induces cell anergy in human Jurkat $T$ cells through modulation of mitogen-activated protein kinases and nuclear factor of activated T-cells signaling with concomitant up-regulation of anergy-associated genes expression. Biol Pharm Bull. 2010:33:40-46.

35. Maus MV, Grupp SA, Porter DL, June CH. Antibody-modified T cells: CARs take the front seat for hematologic malignancies. Blood. 2014;123:2625-35.

36. Baeuerle PA, Reinhardt C. Bispecific T-cell engaging antibodies for cancer therapy. Cancer Res. 2009;69:4941.

37. Fan D, Li W, Yang Y, Zhang X, Zhang Q, Yan Y, et al. Redirection of CD4+ and CD8+ T lymphocytes via an anti-CD3 $\times$ anti-CD19 bispecific antibody combined with cytosine arabinoside and the efficient lysis of patient-derived B-ALL cells. J. Hematol. Oncol. J Hematol Oncol. 2015:8:108.

38. Fan D, Li Z, Zhang X, Yang Y, Yuan $X$, Zhang $X$, et al. AntiCD3Fv fused to human interleukin-3 deletion variant redirected $T$ cells against human acute myeloid leukemic stem cells. J. Hematol. Oncol. J Hematol Oncol. 2015;8:18.

39. Shou P, Chen Q, Jiang J, Xu C, Zhang J, Zheng C, et al. Type I interferons exert anti-tumor effect via reversing immunosuppression mediated by mesenchymal stromal cells. Oncogene. 2016;35:5953-62.
40. Jing W, Chen Y, Lu L, Hu X, Shao C, Zhang Y, et al. Human umbilical cord blood-derived mesenchymal stem cells producing il15 eradicate established pancreatic tumor in syngeneic mice. Mol Cancer Ther. 2014;13:2127-37.

41. Chen X-c, Wang R, Zhao X, Wei Y-q, Hu M, Wang Y-s, et al. Prophylaxis against carcinogenesis in three kinds of unestablished tumor models via IL12-gene-engineered MSCs. Carcinogenesis. 2006;27:2434-41.

42. Kim SM, Lim JY, Park SI, Jeong $\mathrm{CH}$, Oh JH, Jeong M, et al. Gene therapy using TRAIL-secreting human umbilical cord blood-derived mesenchymal stem cells against intracranial glioma. Cancer Res. 2008;68:9614-23.

43. Stoff-Khalili MA, Rivera AA, Mathis JM, Banerjee NS, Moon AS, Hess A, et al. Mesenchymal stem cells as a vehicle for targeted delivery of CRAds to lung metastases of breast carcinoma. Breast Cancer Res Treat. 2007;105:157-67.

44. Yuan X, Zhang Q, Li Z, Zhang X, Bao S, Fan D, et al. Mesenchymal stem cells deliver and release conditionally replicative adenovirus depending on hepatic differentiation to eliminate hepatocellular carcinoma cells specifically. Cancer Lett. 2016;381:85-95.

45. Shi Y, Su J, Roberts Al, Shou P, Rabson AB, Ren G. How mesenchymal stem cells interact with tissue immune responses. Trends Immunol. 2012;33:136-43.

46. Uyttenhove C, Pilotte L, Theate I, Stroobant V, Colau D, Parmentier N, et al. Evidence for a tumoral immune resistance mechanism based on tryptophan degradation by indoleamine 2,3-dioxygenase. Nat Med. 2003;9:1269-74.

47. Ninomiya S, Narala N, Huye L, Yagyu S, Savoldo B, Dotti G, et al. Tumor indoleamine 2,3-dioxygenase (IDO) inhibits CD19-CAR T cells and is downregulated by lymphodepleting drugs. Blood. 2015:125:3905-16.

\section{Submit your next manuscript to BioMed Central and we will help you at every step:}

- We accept pre-submission inquiries

- Our selector tool helps you to find the most relevant journal

- We provide round the clock customer support

- Convenient online submission

- Thorough peer review

- Inclusion in PubMed and all major indexing services

- Maximum visibility for your research

Submit your manuscript at www.biomedcentral.com/submit
Biomed Central 